\title{
What Should Be the Primary Target of "Treat to Target" in Psoriatic Arthritis?
}

\author{
Laura C. Coates (1), Ennio Lubrano (1D), Fabio Massimo Perrotta (D), Paul Emery (i), \\ Philip G. Conaghan (1), and Philip S. Helliwell (1)
}

\begin{abstract}
Objective. Recommendations regarding "treat to target" in psoriatic arthritis (PsA) have stated that the target should be remission or inactive disease. Potential definitions include very low disease activity (VLDA), PsA Disease Activity Score (PASDAS) near remission, Disease Activity Index for PsA (DAPSA) or clinical DAPSA (cDAPSA) remission. Our aim was to investigate the proportion of patients who fulfill these definitions and how much residual active disease remained.

Methods. This analysis used 2 datasets: first, trial data from the Tight Control of PsA (TICOPA) study, which included 206 patients with recent-onset $(<2$ yrs $)$ PsA receiving standard and biological disease-modifying antirheumatic drugs (DMARD); and second, an observational clinical dataset from Italy of patients receiving biological DMARD. Proportions achieving each of the 4 potential targets were calculated in each dataset and comparisons between treatment groups were performed in the TICOPA dataset. Levels of residual disease were established for key clinical domains of PsA.

Results. All measures could differentiate the TICOPA trial treatment groups ( $p<0.03)$. Lower proportions of patients fulfilled the VLDA criteria compared to DAPSA or cDAPSA remission. PASDAS results were different between the cohorts. Residual active disease was low across all definitions although higher levels were seen in DAPSA and cDAPSA compared to VLDA, particularly for psoriasis. In all measures, the proportion with elevated C-reactive protein was similar and low.

Conclusion. VLDA appears the most stringent measure. It ensures that significant active arthritis, enthesitis, and psoriasis are not present, in contrast with DAPSA and PASDAS, in which composite scores can "hide" active disease in some domains. (First Release September 15 2018; J Rheumatol 2019;46:38-42; doi:10.3899/jrheum.180267)
\end{abstract}

Key Indexing Terms: PSORIATIC ARTHRITIS

TREAT TO TARGET

OUTCOME MEASURES

From the Leeds Institute of Rheumatic and Musculoskeletal Medicine, University of Leeds; Leeds Musculoskeletal Biomedical Research Unit, Leeds Teaching Hospitals National Health Service (NHS) Trust, Leeds; Nuffield Department of Orthopaedics, Rheumatology and Musculoskeletal Sciences, University of Oxford, Oxford, UK; Dipartimento di Medicina e Scienze della Salute "Vincenzo Tiberio," Università degli Studi del Molise, Campobasso, Italy.

The TICOPA study was funded by Arthritis Research UK (grant number 18825). L.C. Coates is funded by a National Institute for Health Research Clinician Scientist award. The research is also supported by the National Institute for Health Research (NIHR) Leeds and Oxford Biomedical Research Centre. The views expressed are those of the author(s) and not necessarily those of the NHS, the NIHR, or the Department of Health. The research team acknowledges the support of the Comprehensive Clinical Research Network. Trial registration: The TICOPA trial was registered with ClinicalTrials.gov, number NCT01106079, and the ISCRCTN registry, number ISCRCTN30147736, registered 15 April 2010.

L.C. Coates, MBChB, MRCP (UK), PhD, Leeds Institute of Rheumatic and Musculoskeletal Medicine, University of Leeds, and Leeds Musculoskeletal Biomedical Research Unit, Leeds Teaching Hospitals NHS Trust, and Nuffield Department of Orthopaedics, Rheumatology and Musculoskeletal Sciences, University of Oxford; E. Lubrano, MD, PhD, Associate Professor of Rheumatology and Consultant Rheumatologist, Dipartimento di Medicina e Scienze della Salute "Vincenzo Tiberio," Università degli Studi del Molise; F.M. Perrotta, MD, Clinical Research Fellow,

Dipartimento di Medicina e Scienze della Salute "Vincenzo Tiberio," Università degli Studi del Molise; P. Emery, MA, MD, FRCP, FRCPE, Professor of Rheumatology, Leeds Institute of Rheumatic and Musculoskeletal Medicine, University of Leeds, and Leeds Musculoskeletal
Biomedical Research Unit, Leeds Teaching Hospitals NHS Trust; P.G. Conaghan, MB BS, PhD, FRACP, FRCP, Professor of Musculoskeletal Medicine, Leeds Institute of Rheumatic and Musculoskeletal Medicine, University of Leeds, and Leeds Musculoskeletal Biomedical Research Unit, Leeds Teaching Hospitals NHS Trust; P.S. Helliwell, MA, MD, Senior Lecturer, PhD, Leeds Institute of Rheumatic and Musculoskeletal Medicine, University of Leeds, and Leeds Musculoskeletal Biomedical Research Unit, Leeds Teaching Hospitals NHS Trust.

Address correspondence to Dr. P.S. Helliwell, Leeds Institute of Rheumatic and Musculoskeletal Medicine, University of Leeds, 2nd Floor, Chapel Allerton Hospital, Harehills Lane, Leeds LS7 4SA, UK.

E-mail:p.helliwell@leeds.ac.uk

Accepted for publication June 28, 2018.

In 2013, an international task force published recommendations for treating to target in spondyloarthritis (SpA), including psoriatic arthritis (PsA) ${ }^{1}$. It recommended that "a major treatment target should be clinical remission/inactive disease of musculoskeletal involvement (arthritis, dactylitis, enthesitis, axial disease)" and that "clinical remission/ inactive disease is defined as the absence of clinical and laboratory evidence of significant inflammatory disease activity."

Since then, the Tight Control of PsA (TICOPA) study has shown that treating to target using the minimal disease 
activity (MDA) criteria improves clinical and patient-reported outcomes in PsA despite an increase in drug-related adverse events $^{2}$. However, the MDA criteria encompass both remission and low disease activity and are not analogous with clinical remission/inactive disease.

Potential remission targets in PsA would be very low disease activity (VLDA) defined as meeting all 7 MDA cutpoints $^{3}$, Disease Activity in PsA (DAPSA) remission $(\leq 4)^{4}$, or PsA Disease Activity Score (PASDAS) near remission $(\leq 1.9)^{3}$. VLDA and PASDAS are designed as composite measures of psoriatic disease, while DAPSA is a measure of peripheral arthritis disease activity only. The aim was to investigate which patients fulfill definitions of VLDA and remission, how they compare, and how much residual disease is present.

\section{MATERIALS AND METHODS}

Two datasets were used: TICOPA randomized controlled trial (RCT) ${ }^{2}$, and data from a real-life dataset recruited in Italy (University of Molise) $)^{5}$. The TICOPA trial is a UK multicenter RCT in which 206 adults with early PsA were randomised 1:1 to tight control aiming MDA or standard care ${ }^{2}$.

The Italian dataset included 141 patients with PsA undergoing standard therapy and followup. This analysis included only the 79 who were receiving biological therapy ( 25 adalimumab, 38 etanercept, and 16 golimumab), with or without concomitant disease-modifying antirheumatic drugs with at least 6 months of followup 5 . These patients were $50.6 \%$ male with a mean age (SD) of 53 years (1.3) and mean disease duration (SD) of 6.6 (3.1) years. Appropriate ethical approval was granted for both studies by the Northern and Yorkshire Research Ethics Committee in the UK (TICOPA ref: 07/H0903/72) and by the Comitato Tecnico Scientifico dell'Università degli Studi del Molise, Campobasso, Italy (Italian dataset). All patients gave consent to be involved in the respective studies.

Four potential definitions of remission/inactive disease were used:

1. VLDA ${ }^{3}$ in which all 7 of the MDA cutpoints are met: tender joint count $(\mathrm{TJC}) \leq 1$; swollen joint count $(\mathrm{SJC}) \leq 1$; enthesitis count $\leq 1$; Psoriasis Area and Severity Index (PASI) $\leq 1$; patient global visual analog scale (VAS) $\leq 20 \mathrm{~mm}$; patient pain VAS $\leq 15 \mathrm{~mm}$; and Health Assessment Questionnaire $(\mathrm{HAQ}) \leq 0.5$.

2. DAPSA remission ${ }^{4}$, in which DAPSA $\leq 4[\mathrm{TJC}+\mathrm{SJC}+$ patient global VAS $(\mathrm{cm})+$ pain VAS $(\mathrm{cm})+$ C-reactive protein $(\mathrm{CRP} ; \mathrm{mg} / \mathrm{l})]$.

3. Clinical DAPSA (cDAPSA) remission ${ }^{4}$, in which DAPSA $\leq 4(\mathrm{TJC}+$ $\mathrm{SJC}+$ patient global VAS + pain VAS).

4. Near remission in the PASDAS ${ }^{3}$ where PASDAS $\leq 1.9$. PASDAS is calculated as $[((0.18 \sqrt{ }$ physician global VAS $)+(0.159 \sqrt{ }$ patient global VAS $)$ $-(0.253 \times \sqrt{ } \mathrm{SF}-36-\mathrm{PCS})+(0.101 \times \mathrm{LN}(\mathrm{SJC}+1))+(0.048 \times \mathrm{LN}(\mathrm{TJC}+$ $1))+(0.23 \times \mathrm{LN}($ Leeds enthesitis index +1$))+(0.37 \mathrm{LN}$ (tender dactylitis count +1$))+(0.102 \times \mathrm{LN}(\mathrm{CRP}+1))+2] \times 1.5$. SF-36 is the Medical Outcomes Study Short Form-36 questionnaire; PCS is the physical component summary of the SF-36; and LN is logarithm.

Statistical methods. Full data to calculate the composite PsA disease activity index were not available and so this definition was not included. In the datasets, the PASDAS was calculated using an imputed measure because SF-36 was not collected in the trial ${ }^{6}$. Proportions achieving each criterion were calculated. Only patients with full data were included in the comparison. Comparisons between treatment groups in the TICOPA study were performed using chi-square test. The agreement between the tested definitions was established using $2 \times 2$ tables, Cohen's $\kappa$ percentage exact agreement (PEA). The proportion of residual disease was established for key clinical domains of PsA (peripheral arthritis, enthesitis, psoriasis, dactylitis) and levels of systemic inflammation, as measured by CRP, were assessed.

\section{RESULTS}

At the end of the TICOPA study (48 weeks), 50 patients (24.3\%) were in DAPSA remission, $56(27.2 \%)$ were in cDAPSA remission, 49 (23.8\%) were in PASDAS remission, and $27(13.1 \%)$ met VLDA. However, missing data precluded calculation for DAPSA in 34 patients, cDAPSA in 23, and VLDA in 24. For fair comparison, subsequent statistics are based on those with full data for all measures $(\mathrm{n}=170)$.

The difference in proportion of patients achieving all definitions was significant between tight control and standard care $(\mathrm{p}<0.03$, Table 1$)$ in the TICOPA study. In Italy, at 6 months of followup, 18 patients $(22.7 \%)$ were in DAPSA remission, $22(27.8 \%)$ were in cDAPSA remission, 13 $(16.5 \%)$ were in PASDAS remission, and 15 (18.9\%) were in VLDA.

There was a very high agreement found between DAPSA and cDAPSA remission (Cohen's $\kappa 0.931,95 \%$ CI $0.87-0.99, \mathrm{p}<0.001$ in TICOPA, $0.86,95 \%$ CI $0.76-0.97$ in Italy), reflecting their similar components. The agreement was lower but still moderate to substantial between VLDA and both DAPSA remission definitions; VLDA agreement with both DAPSA and cDAPSA remission in TICOPA was 0.57 (95\% CI $0.43-0.71$ ) and 0.52 (95\% CI 0.38-0.65), respectively, and in Italy the $\kappa$ was 0.66 (95\% CI $0.48-0.83$ ) and 0.60 (95\% CI 0.42-0.77), respectively. PASDAS remission showed similar strength agreement with all other measures [in TICOPA - DAPSA rem $0.66(95 \%$ CI $0.53-0.78)$, cDAPSA rem 0.60 (95\% CI 0.47-0.73), VLDA 0.58 (95\% CI 0.44-0.73); in Italy - DAPSA rem 0.66 (95\% CI 0.45-0.87), cDAPSA rem 0.55 (95\% CI 0.37-0.72), and VLDA 0.56 (95\% CI 0.33-0.83)].

At an individual patient level, there was good agreement between VLDA and DAPSA/cDAPSA remission with a PEA of $84.7 / 81.8 \%$, respectively, in TICOPA and 86.1/83.4 in Italy. However, this was partially driven by the numbers not fulfilling either target. In TICOPA, there were 25 people in DAPSA remission and 30 in cDAPSA remission who did not meet VLDA. For most of these, the patients fulfilled 6 of the criteria with residual disease activity in 1 domain (skin: $\mathrm{n}=16$, enthesitis: $\mathrm{n}=5$, tender joints: $\mathrm{n}=2$, and active swollen joints: $\mathrm{n}=3$ ). Three patients did not fulfill 2 domains (swollen joints/skin: $\mathrm{n}=1$, tender joints/skin: $\mathrm{n}=1$, and tender joints/HAQ: $n=1$ ). One patient only fulfilled $3 / 7$ MDA domains. In those cases, disease activity was over the VLDA threshold for the individual measures but if the other DAPSA components were low (e.g., 2 swollen but non-tender joints), then DAPSA remission could still be achieved. In contrast, only 1 person was not in DAPSA remission (DAPSA score 4.8) but in VLDA. In Italy, there were 6 people in DAPSA remission and 10 in cDAPSA remission who were not in VLDA. The residual disease activity for both groups was in 1 domain, for those in DAPSA remission but not VLDA (skin $n=5, \mathrm{HAQ}=1$ ) and those in cDAPSA (skin

Personal non-commercial use only. The Journal of Rheumatology Copyright (c) 2019. All rights reserved. 
Table 1. Complete case analysis of TICOPA.

\begin{tabular}{lcccc}
\hline Target & Tight Control, $\mathrm{n}(\%)$ & Standard Care, $\mathrm{n}(\%)$ & Chi-squared & $\mathrm{p}$ \\
\hline DAPSA remission & $35(20.6)$ & $15(8.8)$ & 8.84 & 0.004 \\
cDAPSA remission & $39(22.9)$ & $16(9.4)$ & 11.2 & 0.001 \\
VLDA & $21(12.4)$ & $5(2.9)$ & 9.94 & 0.002 \\
PASDAS remission & $28(16.5)$ & $13(7.6)$ & 5.50 & 0.021 \\
\hline
\end{tabular}

TICOPA: Tight Control of Psoriatic Arthritis study; DAPSA: Disease Activity Index for Psoriatic Arthritis; cDAPSA: clinical DAPSA; VLDA: very low disease activity; PsA: psoriatic arthritis; PASDAS: PsA Disease Activity Score.

$\mathrm{n}=5, \mathrm{HAQ}=2$, pain VAS $=3$ ). In contrast, only $3 / 2$ people were not in DAPSA/cDAPSA remission but were in VLDA.

When considering PASDAS remission, scores showed good agreement with PEA of $86.4 \%$ for VLDA and $86.5 / 83.5 \%$ for DAPSA/cDAPSA remission. In Italy, PEA for VLDA and DAPSA/cDAPSA remission were 86.8/93.4/89.5\%, respectively. Again, these are driven by patients fulfilling neither target. Using the TICOPA dataset, VLDA seems more stringent, with only 4 patients in VLDA with a PASDAS score of $>1.9$ but 19 patients in PASDAS remission who did not meet VLDA. When comparing DAPSA and PASDAS, it was more common for patients to be in DAPSA remission but not PASDAS remission $(n=16$ for DAPSA and $n=21$ for cDAPSA) than it was for patients to be in PASDAS remission without meeting DAPSA ( $\mathrm{n}=7$ for DAPSA remission and $\mathrm{n}=7$ for cDAPSA remission).

Levels of residual active disease in patients meeting the remission/VLDA criteria are shown in Table 2 and Table 3. All definitions had similar proportions of residual disease, except for residual psoriasis in TICOPA, which was excluded

Table 2. Residual disease activity in remission and VLDA definitions for 170 patients with full data in the TICOPA trial.

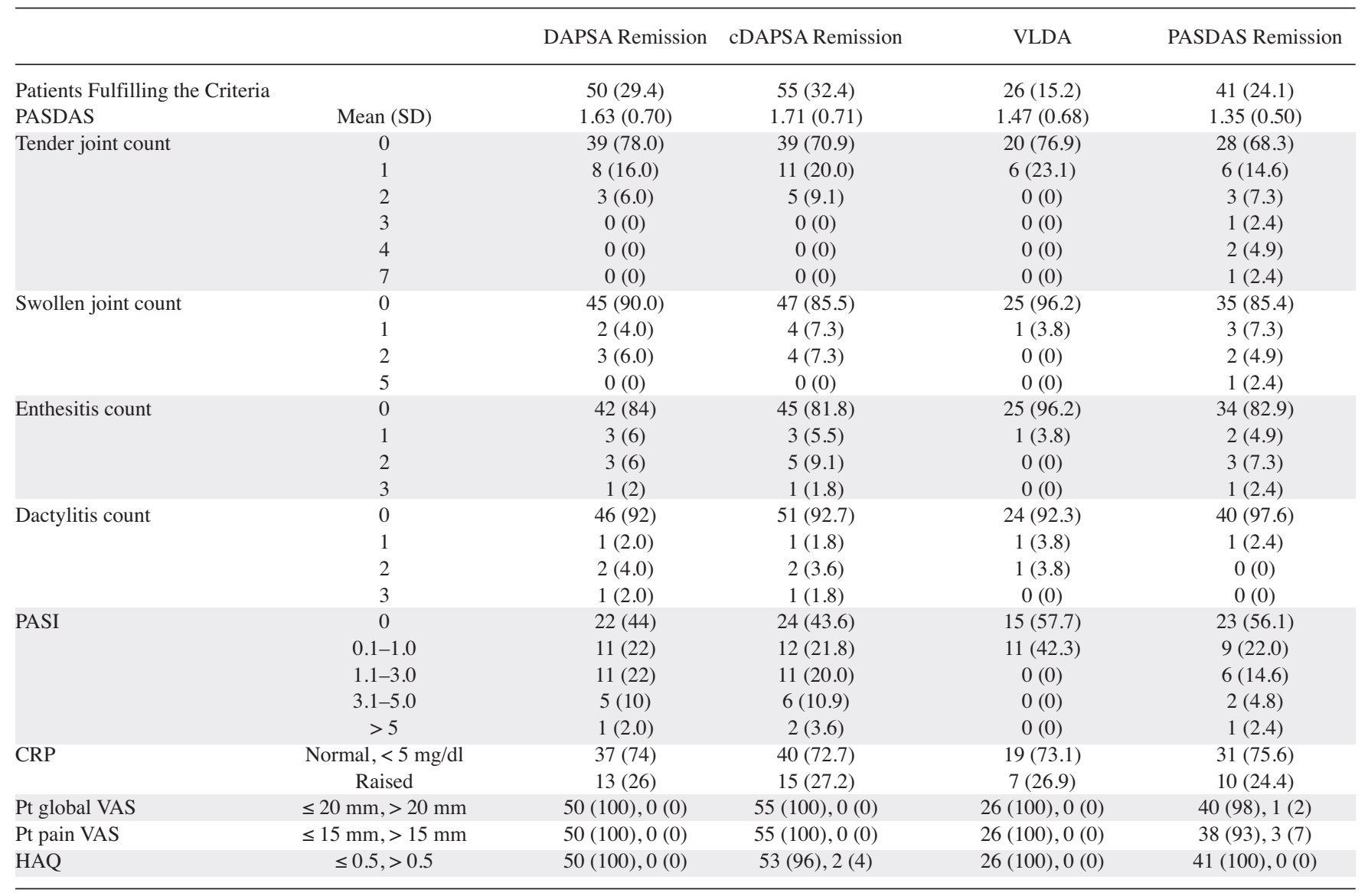

Data are n (\%), except where indicated. TICOPA: Tight Control of Psoriatic Arthritis study; CRP: C-reactive protein; DAPSA: Disease Activity in Psoriatic Arthritis; HAQ: Health Assessment Questionnaire, PASI: Psoriasis Area and Severity Index; PASDAS: Psoriatic Arthritis Disease Activity Score; VAS: visual analog scale; VLDA: very low disease activity; cDAPSA: clinical DAPSA. 
Table 3. Residual disease activity in remission and VLDA definitions in real-life dataset.

\begin{tabular}{|c|c|c|c|c|c|}
\hline & & DAPSA Remission & cDAPSA Remission & VLDA & PASDAS Remission \\
\hline \multicolumn{2}{|c|}{ Patients Fulfilling the Criteria } & $18(22.8)$ & $22(27.8)$ & $15(19.0)$ & $13(16.5)$ \\
\hline PASDAS & Mean (SD) & $1.45(0.76)$ & $1.66(0.87)$ & $1.68(0.98)$ & $1.06(0.45)$ \\
\hline \multirow[t]{3}{*}{ Tender joint count } & 0 & $13(72.2)$ & $16(72.7)$ & $9(60)$ & $13(100)$ \\
\hline & 1 & $5(27.6)$ & $6(27.3)$ & $6(40)$ & 0 \\
\hline & 2 & 0 & 0 & 0 & 0 \\
\hline \multirow[t]{3}{*}{ Swollen joint count } & 0 & $17(94.5)$ & $21(95.4)$ & $14(93.3)$ & $12(92)$ \\
\hline & 1 & $1(5.5)$ & $1(4.5)$ & $1(6.7)$ & $1(8)$ \\
\hline & 2 & 0 & 0 & 0 & 0 \\
\hline \multirow[t]{3}{*}{ Enthesitis count } & 0 & $17(94.5)$ & $19(86.3)$ & $13(86.7)$ & $11(85)$ \\
\hline & 1 & $1(5.5)$ & $2(9.0)$ & $2(13.3)$ & $2(15)$ \\
\hline & 2 & 0 & $1(4.5)$ & 0 & 0 \\
\hline \multirow[t]{4}{*}{ PASI } & $0-0.3$ & $11(61.1)$ & $14(63.6)$ & $12(80)$ & $9(69)$ \\
\hline & $0.4-0.6$ & $2(11.1)$ & $2(9.0)$ & $2(13.3)$ & $1(8)$ \\
\hline & $0.7-1$ & 0 & $1(4.5)$ & $1(6.7)$ & 0 \\
\hline & $>1$ & $5(27.7)$ & $5(22.7)$ & 0 & $3(23)$ \\
\hline \multirow[t]{2}{*}{ CRP } & Normal, $<5$ mg/dl & $17(94.4)$ & $20(91)$ & $14(93.3)$ & $13(100)$ \\
\hline & Raised & $1(5.5)$ & $2(9.0)$ & $1(6.7)$ & 0 \\
\hline Pt global VAS & $\leq 20 \mathrm{~mm},>20 \mathrm{~mm}$ & $18(100), 0(0)$ & $22(100), 0(0)$ & $15(100), 0(0)$ & $13(100), 0(0)$ \\
\hline Pt pain VAS & $\leq 15 \mathrm{~mm},>15 \mathrm{~mm}$ & $18(100), 0(0)$ & $20(90.9), 2(9.1)$ & $15(100), 0(0)$ & $13(100), 0(0)$ \\
\hline HAQ & $\leq 0.5,>0.5$ & $17(94.5), 1 \quad(5.5)$ & $20(90.9), 2(9.1)$ & $15(100), 0(0)$ & $13(100), 0(0)$ \\
\hline
\end{tabular}

Data are n (\%), except where indicated. CRP: C-reactive protein; DAPSA: Disease Activity in Psoriatic Arthritis; cDAPSA: clinical DAPSA; HAQ: Health Assessment Questionnaire; PASI: Psoriasis Area and Severity Index; PASDAS: Psoriatic Arthritis Disease Activity Score; VAS: visual analog scale; VLDA: very low disease activity.

by VLDA but highest in DAPSA remission (34\% with PASI $>1$ ) and moderate in PASDAS remission (22\% with PASI $>1$ ). All definitions had similar proportions of patients with raised CRP levels despite it not being included in either VLDA or cDAPSA definitions.

\section{DISCUSSION}

This analysis reports the first comparison of potential remission targets in PsA, to our knowledge. All were able to differentiate significantly between treatment groups in the RCT. While similar proportions of patients fulfilled the DAPSA and cDAPSA remission, fewer people fulfilled the VLDA criteria, suggesting that they are more stringent. The differences were predominantly due to active psoriasis, but in a few patients, residual arthritis and enthesitis were seen in DAPSA or cDAPSA remission in the TICOPA cohort. The PASDAS remission criteria showed variable results, with higher proportions achieving the criteria in TICOPA compared to VLDA but with the highest level of residual arthritis. While in the Italian cohort, PASDAS showed similar results to VLDA, with low levels of residual disease.

This retrospective analysis provides important data comparing different treatment targets in PsA, allowing direct comparison. Unfortunately, we do not have health state "anchor" questions for either patient or physician for comparison. Further work is needed to establish the optimal level of disease control, balancing beneficial longterm outcome with potential risk of therapy and incorporating physician and patient opinion. Additional analysis in other datasets would aid this debate by providing evidence on whether treating to remission targets such as VLDA is superior to treating to MDA as in the TICOPA trial regarding disease effect and radiographic outcomes and how this affects the prevalence of treatment-related adverse events.

PASDAS and VLDA were developed as multidimensional measures including different domains of psoriatic disease to reflect overall disease activity in a multisystem disease. In contrast, the DAPSA focuses particularly on peripheral arthritis and does not include other domains, because the developers wanted to devise a unidimensional measure that would assess only 1 element of the disease ${ }^{7}$. This explains some of the mismatch between DAPSA remission and VLDA/PASDAS when residual active skin disease was present. This highlights the need for a separate skin measure to also be used alongside DAPSA if this is chosen over a multidimensional definition, to ensure that face validity is maintained for these patients. Interestingly, residual dactylitis activity was identified in 3 patients, although the joint counts in the measures should have identified this. Further instructions may be useful to highlight that tender/swollen dactylitic digits should also be recorded in the joint counts.

Examining residual active disease highlighted a potential issue with DAPSA and PASDAS scores, as composites, compared to the approach of VLDA, where individual items are assessed separately rather than summed together. Because both DAPSA and PASDAS sum their scores into 1 final number, the balance of scores for each domain can "hide" active disease in some domains. In both the DAPSA and the

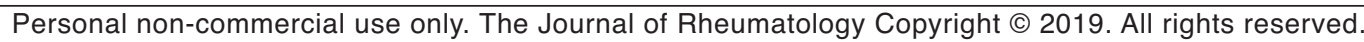


PASDAS, higher levels of residual active disease were seen, particularly in the TICOPA cohort. For PASDAS this may reflect the fact that the patient-reported domains are more heavily weighted than clinical measurements. In VLDA, because all items are required to meet an individual cutpoint, residual active disease is limited. While fewer people fulfill this definition, the lower levels of residual disease activity increase its face validity for the concept of "remission."

Two of the scores require a CRP (DAPSA and PASDAS; Table 4). These data suggest that the inclusion of a laboratory marker is unnecessary because a similar proportion of patients have raised CRP levels in all definitions. This has a practical advantage, making target assessment easier in clinical practice. VLDA includes a measure of function because the items within it were taken from the PsA core $\operatorname{set}^{8}$, following the development methodology of the RA MDA criteria $^{9}$. This is a potential limitation of the VLDA because HAQ can be affected by irreversible damage as well as disease activity. In these cohorts, however, very few patients failed to achieve VLDA because of HAQ alone. For patients with significant damage, it may be possible to achieve only 6 of the 7 cutpoints (excluding HAQ), but they would still be classified as being in MDA, thus fulfilling the treat-to-target recommendations using the alternative target ${ }^{7}$.

All these measures require patient- and physician-reported items. PASDAS is the most involved, requiring a complex formula including joint, enthesitis, and dactylitis counts, physician's global assessment, patient's global assessment, SF-36 PCS, and CRP. This may be less feasible for clinical use, although apps or spreadsheets can be used. Both DAPSA and VLDA scores are simpler to calculate, both requiring a full 66/68 joint count, and a patient pain and global VAS. In addition, MDA requires assessment of enthesitis and skin. While this does slightly increase the time for assessment,

Table 4. Comparison of individual measures contained in the composite targets.

\begin{tabular}{lcccc}
\hline & DAPSA & cDAPSA & VLDA & PASDAS \\
\hline TJC & Yes & Yes & Yes & Yes \\
SJC & Yes & Yes & Yes & Yes \\
Pt global VAS & Yes & Yes & Yes & Yes \\
Pt pain VAS & Yes & Yes & Yes & No \\
CRP & Yes & No & No & Yes \\
Enthesitis & No & No & Yes & Yes \\
Psoriasis rash & No & No & Yes & No \\
HAQ & No & No & Yes & No \\
Dactylitis & No & No & No & Yes \\
Physician's global VAS & No & No & No & Yes \\
SF-36 PCS & No & No & No & Yes \\
& & & &
\end{tabular}

DAPSA: Disease Activity in Psoriatic Arthritis; cDAPSA: clinical DAPSA; VLDA: very low disease activity; PASDAS: Psoriatic Arthritis Disease Activity Score; TJC: tender joint count; SJC: swollen joint count; VAS: visual analog scale; CRP: C-reactive protein; HAQ: Health Assessment Questionnaire; SF-36: Medical Outcomes Study Short Form-36 questionnaire; PCS: SF-36 physical component summary. enthesitis can be assessed alongside the joint examination (Leeds enthesitis index is only 6 sites), and the skin item is quickly classified as $\leq 3$ palms of body area covered or greater.

The weighted PASDAS retains more dependence on patient-reported outcomes and less on clinical measures, and like the DAPSA, depends on a laboratory biomarker that may decrease feasibility. Both cDAPSA and VLDA can be performed relatively easily in routine clinical practice and perform well without the inclusion of an inflammatory blood marker. Neither needs a complex formula, and in fact there is a significant overlap between their items. While both are associated with improved outcomes, VLDA is a more stringent measure in keeping with the concept of remission; the brief assessment of enthesitis and skin disease ensure that other domains outside peripheral arthritis are not missed, and its individual cutpoints for different domains ensure very low levels of residual active disease across all key domains.

Our study compared different remission definitions and has shown that all could differentiate between treatment groups in a clinical trial (TICOPA), but VLDA was associated consistently with the lower levels of residual disease activity, supporting its face validity as a definition of remission in PsA.

\section{REFERENCES}

1. Smolen JS, Braun J, Dougados M, Emery P, Fitzgerald O, Helliwell $\mathrm{P}$, et al. Treating spondyloarthritis, including ankylosing spondylitis and psoriatic arthritis, to target: recommendations of an international task force. Ann Rheum Dis 2014;73:6-16.

2. Coates LC, Moverley AR, McParland L, Brown S, Navarro-Coy N, O'Dwyer JL, et al. Effect of tight control of inflammation in early psoriatic arthritis (TICOPA): A UK multicentre, open-label, randomised controlled trial. Lancet 2015;386:2489-98.

3. Coates LC, Helliwell PS. Defining low disease activity states in psoriatic arthritis using novel composite disease instruments. J Rheumatol 2016;43:371-5.

4. Schoels MM, Aletaha D, Alasti F, Smolen JS. Disease activity in psoriatic arthritis (PsA): Defining remission and treatment success using the DAPSA score. Ann Rheum Dis 2016;75:811-8.

5. Perrotta FM, Marchesoni A, Lubrano E. Minimal disease activity and remission in psoriatic arthritis patients treated with anti-TNF- $\alpha$ drugs. J Rheumatol 2016;43:350-5.

6. Coates LC, Mahmood F, Emery P, Conaghan PG, Helliwell PS. The dynamics of response as measured by multiple composite outcome tools in the tight control of inflammation in early psoriatic arthritis (TICOPA) trial. Ann Rheum Dis 2017;76:1688-92.

7. Smolen JS, Schöls M, Braun J, Dougados M, FitzGerald O, Gladman DD, et al. Treating axial spondyloarthritis and peripheral spondyloarthritis, especially psoriatic arthritis, to target: 2017 update of recommendations by an international task force. Ann Rheum Dis 2018;77:3-17.

8. Gladman DD, Mease PJ, Strand V, Healy P, Helliwell PS, Fitzgerald $\mathrm{O}$, et al. Consensus on a core set of domains for psoriatic arthritis. J Rheumatol 2007;34:1167-70.

9. Wells GA, Boers M, Shea B, Brooks PM, Simon LS, Strand CV, et al. Minimal disease activity for rheumatoid arthritis: a preliminary definition. J Rheumatol 2005;32:2016-24. 